\title{
Tratamento para mulheres inférteis com Síndrome dos Ovários Policísticos (SOP)
}

\author{
Treatment for infertile women with Polycystic Ovarian Syndrome (PCOS)
}

Tratamiento para mujeres infértiles con Síndrome de Ovario Poliquístico (SOP)

Ana Elise de Souza Barros Pereira ${ }^{1 *}$, Anne Caroline Rogienfisz Mendes ${ }^{2}$, Beatriz Davantel Klaus $^{3}$, Carolina Souto Azevedo ${ }^{4}$, Gabriela Wosniak Cavaletti ${ }^{5}$, Laíze Vessali de Almeida Lopes $^{6}$, Milagres Araújo Nascimento ${ }^{7}$, Rhayssa Vasconcelos Leitão ${ }^{4}$, Tainara Sales Miranda ${ }^{8}$, Raquel Meirelles Gaspar Coelho Guimarães ${ }^{9}$.

\section{RESUMO}

Objetivo: Descrever o conhecimento sobre as terapias medicamentosas para tratar as mulheres que possuem infertilidade por causa da Síndrome do Ovário Policístico (SOP). Revisão bibliográfica: O letrozol é a droga de primeira linha para indução de ovulação no tratamento de mulheres com SOP. O citrato de clomifeno fica reservado para os casos em que não houver possibilidade de uso de letrozol como primeira linha. A associação da metformina está recomendada nos casos de resistência insulínica associada a SOP ou má resposta aos indutores da ovulação. Em relação ao uso de gonadotrofinas induzem a ovulação, há evidências de taxas superiores de gravidez clínica, gravidez em curso e nascidos vivos da indução da ovulação com gonadotrofinas quando comparada ao CC, no entanto, devido seu maior custo, e maior risco de hiperestimulo ovariano, esse medicamento entra como tratamento de segunda linha. Outra opção terapêutica seria a utilização de mio-inositol mas as evidencias são de baixa qualidade. Por fim, a fertilização in vitro também faz parte do tratamento, mas os artigos demonstram que apenas se tratamentos anteriores não funcionarem. Considerações finais: $O$ tratamento de SOP é multifatorial, e não há um tratamento único para todas as pacientes. Novos estudos são necessários.

Palavras-chave: Síndrome do ovário policístico, Infertilidade, Tratamento.

\begin{abstract}
Objective: To describe the knowledge about drug therapies to treat women who have infertility because of Polycystic Ovary Syndrome (PCOS). Bibliographic review: Letrozole is the first-line drug for inducing ovulation in the treatment of women with PCOS. Clomiphene citrate is reserved for cases where there is no possibility of using letrozole as a first line. The combination of metformin is recommended in cases of insulin resistance associated with PCOS or poor response to ovulation inducers. Regarding the use of gonadotropins that induce ovulation, there is evidence of higher rates of clinical pregnancy, ongoing pregnancy and live births of ovulation induction with gonadotropins when compared to CC, however, due to its higher cost, and greater risk of ovarian hyperstimulation this medication is used as a second-line treatment. Another therapeutic option would be the use of myo-inositol but the evidence is of low quality. Finally, in vitro fertilization is also part of the treatment, but the articles demonstrate that only if previous treatments do not work. Final considerations: The treatment of PCOS is multifactorial, and there is no single treatment for all patients. Further studies are needed.
\end{abstract}

Keywords: Polycystic ovary syndrome, Infertility, Treatment.

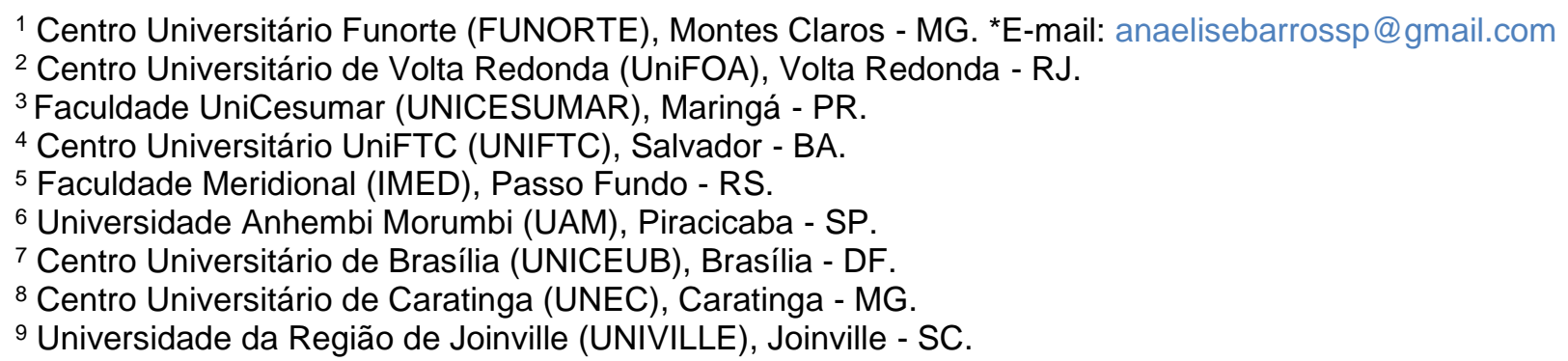




\section{RESUMEN}

Objetivo: Describir el conocimiento sobre terapias farmacológicas para el tratamiento de mujeres que padecen infertilidad por síndrome del ovario poliquístico (SOP). Revisión bibliográfica: El letrozol es el fármaco de primera línea para inducir la ovulación en el tratamiento de mujeres con SOP. El citrato de clomifeno se reserva para los casos en los que no existe la posibilidad de utilizar letrozol como primera línea. La combinación de metformina se recomienda en casos de resistencia a la insulina asociada con SOP o mala respuesta a los inductores de la ovulación. En cuanto al uso de gonadotropinas que inducen la ovulación, existe evidencia de mayores tasas de embarazo clínico, embarazo en curso y nacidos vivos de inducción de la ovulación con gonadotropinas en comparación con CC, sin embargo, debido a su mayor costo y mayor riesgo de hiperestimulación ovárica este medicamento se utiliza como tratamiento de segunda línea. Otra opción terapéutica sería el uso de mioinositol pero la evidencia es de baja calidad. Por último, la fecundación in vitro también forma parte del tratamiento, pero los artículos lo demuestran solo si los tratamientos previos no funcionan. Consideraciones finales: el tratamiento del SOP es multifactorial y no existe un tratamiento único para todos los pacientes. Se necesitan más estudios.

Palabras clave: Síndrome del Ovario Poliquístico, Esterilidad, Tratamiento.

\section{INTRODUÇÃO}

A Síndrome dos Ovários Policísticos (SOP) é um dos principais distúrbios endócrino-ginecológicos e atinge cerca de $6 \%$ a $18 \%$ das mulheres em idade reprodutiva, representando uma das principais causas de infertilidade (CONCHA FC, et al., 2017). Esta patologia gera diversas implicações na vida da mulher, podendo acometer além do sistema reprodutor feminino e endócrino, também o sistema cardiovascular, a pele e muitas vezes a qualidade de vida e a sensação de bem-estar dessas mulheres (HOFFMAN B, 2014).

Sua etiologia ainda é desconhecida, porém acredita-se que sua origem é multifatorial e envolva alterações genéticas, metabólicas e endócrinas e suas respectivas associações às condições ambientais. As alterações genéticas podem envolver a alteração de genes relacionados com a síntese, ação e regulação de androgênios. Os fatores metabólicos e endócrinos preponderantes associada a SOP são o aumento da resistência periférica à insulina, e alteração da resposta hipofisária para a secreção do Hormônio Luteinizante (LH) e do Hormônio Folículo Estimulante (FSH) (HOFFMAN B, 2014; FREITAS LA, et al., 2016).

Dessa forma, os principais sintomas relacionados a SOP são irregularidade menstrual e sinais de hiperandrogenismo como acne, excesso de pêlos e alopécia. No entanto, faz-se sempre necessário o diagnóstico diferencial baseado na imaturidade do eixo hipotálamo-hipofisário-ovariano e de distúrbios psíquicos. Assim, o diagnóstico só será firmado após exclusão de afecções, como: disfunção da tireóide, hiperprolactinemia, tumor ovariano ou da suprarrenal, defeitos de síntese da suprarrenal e síndrome de Cushing (FEBRASGO, 2018).

Diante das consequências geradas pela SOP, que impactam na qualidade de vida, a terapêutica é indicada individualmente, considerando as necessidades de cada mulher, bem como sinais e sintomas. $O$ objetivo do tratamento é corrigir as anormalidades hormonais, aliviar os sintomas e melhorar a fertilidade, (MOURA HHG, et al., 2011).

A infertilidade, que se caracteriza pela ausência de gestação após 12 meses de tentativas sem a utilização de quaisquer métodos contraceptivos, é uma das principais consequências da SOP e leva inúmeras mulheres a procurarem tratamento, visto que essa síndrome é responsável por cerca de $80 \%$ dos casos de infertilidade anovulatória (FEBRASGO, 2018). No entanto, não existe um tratamento modelo para a infertilidade ocasionada por SOP. Esse varia desde mudanças no estilo de vida, terapias medicamentosas e técnicas de reprodução assistida (SANTANA LF, et al., 2008).

O presente trabalho tem por objetivo discutir os tipos de tratamentos para mulheres inférteis que possuem SOP, destacando os resultados dessas terapêuticas. 


\section{REVISÃO BIBLIOGRÁFICA}

\section{Definição}

A SOP é uma das endocrinopatias ginecológicas mais frequentes em mulheres em idade reprodutiva e, há alguns anos, era vista apenas como um problema do órgão reprodutor feminino. Atualmente, sabe-se que é uma desordem endócrino-metabólica com envolvimento de todo o organismo da mulher e estima-se que essa síndrome acometa entre 6 a 18\% das mulheres em idade reprodutiva (SOUSA RL, et al., 2013). É caracterizada por manifestações clínicas que incluem resistência à insulina, hiperandrogenismo e irregularidade menstrual ou amenorreia (RESENDE LOT, et al., 2010; SANTOS RM e ÁLVARES ACM, 2018).

A SOP tem origem multifatorial que envolve alterações genéticas, fatores endócrinos, metabólicos e a associação de todos esses ao ambiente. A alteração genética encontrada é um tipo de herança poligênica, onde os genes envolvidos no desenvolvimento da SOP estão relacionados com a síntese, ação e regulação de androgênios, que são hormônios responsáveis pelo desenvolvimento e prosseguimento das características sexuais masculinas, além de genes relacionados à resistência insulínica. Dessa forma, as pacientes geralmente cursam com uma subexpressão dos receptores de insulina em tecidos metabólicos, dentre eles o principal o tecido adiposo, e um aumento da expressão no tecido ovariano, justificando, assim, a resistência insulínica periférica e a hipersecreção de androgênios (SANTANA LF, et al., 2008; FEBRASGO, 2018).

Dentre as condições endócrinas relacionadas, ocorre uma alteração nos pulsos do Hormônio Liberador de Gonadotrofinas $(\mathrm{GnRH})$ pelo hipotálamo e essa mudança da secreção de GnRH gera uma estimulação anormal na hipófise. Com o aumento da amplitude dos pulsos de GnRH ocorre uma hipersecreção da liberação do LH, condição bastante característica dessa síndrome, e consequentemente, secreção baixa ou no limiar da normalidade do FSH (FREITAS LA, et al., 2016).

O aumento do LH leva a uma hiperatividade das células da teca, responsáveis por produzir androgênios em excesso, especialmente a testosterona, sem que haja a conversão adequada desse androgênio em estradiol. Este fato, juntamente com os baixos níveis de $\mathrm{FSH}$, gera um microambiente estrogênico, impossibilitando, assim, o desenvolvimento dos folículos até estágios mais maduros e isso é o que caracteriza a morfologia policística do ovário, facilmente visualizada pelo exame ecográfico. Assim, tem-se um distúrbio no desenvolvimento do folículo e anovulação (SPEROFF L, et al., 1995; FEBRASGO, 2018).

Sabe-se, ainda, que as células da teca em mulheres com SOP possuem sensibilidade aumentada à insulina, o que estimula, ainda mais, a produção de androgênios. O excesso de androgênios age no fígado diminuindo a produção da Globulina Ligadora de Hormônios Sexuais (SHBG) e aumentando a concentração de androgênios livres. Ocorre, então, aumento da resistência periférica à insulina, aumento da concentração de glicose sanguínea e aumento da produção de insulina, que consequentemente vai agir nas células da teca, gerando um ciclo de autoperpetuação. Logo, mulheres portadoras de SOP possuem mais chances de apresentar quadros de resistência insulínica e hiperinsulinemia compensatória, independentemente de estar obesa ou não, culminando em maior probabilidade de desenvolvimento de diabetes mellitus, síndromes metabólicas e doenças cardiovasculares (SANTANA LF, et al., 2008; RESENDE LOT, et al., 2010; FREITAS LA, et al., 2016).

\section{Critérios Diagnósticos}

Com o intuito de auxiliar no diagnóstico dessa patologia, em 2003 foram propostos os Critérios de Rotterdam, em que, a partir da apresentação de dois entre os três critérios estabelecidos, tais como, oligo/anovulação, hiperandrogenismo e ovário com volume acima de $10 \mathrm{~cm}^{3}$ ou com 12 ou mais folículos com diâmetro médio entre $2-9 \mathrm{~mm}$, confirmados por ultrassonografia, fazendo-se a confirmação diagnóstica (Rotterdam ESHRE/ASRM-Sponsored PCOS Consensus Workshop Group, 2004) (MOURA HHG, et al., 2011).

No entanto, esse critério utilizado por mais de décadas vem sendo questionado ao longo dos anos, por não abordar separadamente adolescentes e mulheres em maturidade ginecológica, por não fazer distinção entre as apresentações clinicas e não levar em consideração etnia e fatores de risco para SOP. Sabe-se que a apresentação da SOP varia de acordo com a etnia e em populações de alto risco. Mulheres indígenas, por exemplo, possuem maior prevalência e complicações relacionadas a SOP (TEED HJ, et al, 2018). 
O Guideline Internacional de SOP, publicado em 2018, endossa os critérios diagnósticos de Rotterdam para SOP em adultos, frisando que quando houver ciclos menstruais irregulares e hiperandrogenismo juntos, a ultrassonografia não é necessária para o diagnóstico. Esse guideline também reforça as recomendações do workshop do $(\mathrm{NIH})$, de 2012, que identifica quatro fenótipos específicos das pacientes com SOP e explica que os mesmos devem ser relatados explicitamente em todas as pesquisas para facilitar a identificação das características de cada paciente e saber, no futuro, as reais implicações desses fenótipos nos desfechos clínicos de SOP (TEED HJ, et al, 2018).

Os fenótipos descritos para SOP são: excesso de androgênio associado a disfunção ovulatória e morfologia ovariana policística (fenótipo A), excesso de andrógeno associado apenas a disfunção ovulatória (fenótipo B), excesso de andrógeno associado apenas a morfologia do ovário policístico (fenótipo C), disfunção ovulatória associada apenas a morfologia ovariana policística (Fenótipo D) (TEED HJ, et al, 2018).

Exclusão de doença tireoidiana através da dosagem do hormônio estimulador da tireoide (TSH), de hiperprolactinemia, com a dosagem da prolactina (PRL) e de hiperplasia adrenal congênita não clássica, através da dosagem de 17-hidroxi progesterona, é recomendada como avaliação adicional em pessoas com amenorreia e características clínicas mais graves, buscando também descartar hipogonadismo hipogonadotrópico, Doença de Cushing ou tumores produtores de andrógenos. A diretriz visa facilitar o diagnóstico oportuno e apropriado para mulheres com SOP, evitando o excesso de diagnóstico, especialmente em adolescentes. As recomendações específicas de relevância aqui incluem: a ultrassonografia não é mais recomendada para diagnóstico em pessoas com menos de 8 anos de menarca; e mulheres jovens com fatores de risco, quando o diagnóstico não for claro, devem ser acompanhadas (TEED $\mathrm{HJ}$, et al, 2018).

Em relação à anovulação, importante lembrar que disfunções ovulatorias ocorrem mesmo na ausência de amenorréia ou em ciclos regulares, e que em caso de necessidade de confirmação diagnostica a dosagem de progesterona na segunda fase do ciclo pode ser utilizada (TEED HJ, et al, 2018).

O hiperandrogenismo, caracterizado pelo aumento de andrógenos no sangue, geralmente acarreta o desenvolvimento de hirsutismo, acne, alopecia, distúrbios menstruais e disfunções ovulatórias (YARAK S, et al., 2005). Seu diagnostico laboratorial pode ser realizado através do cálculo da testosterona livre, do índice de androgênio livre ou da testosterona biodisponível. Já testes disponíveis para avaliação direta de testosterona livre, possuem baixa sensibilidade, acurácia e precisão e não devem ser utilizados (TEED HJ, et al, 2018).

As dosagens de androstenediona e sulfato de desidroepiandrosterona (DHEAS) podem ser considerados se a testosterona total ou livre não estiver elevada; no entanto, eles fornecem informações adicionais limitadas no diagnóstico de SOP. Importante frisar que a avaliação bioquímica do hiperandrogenismo não é possível em mulheres sob contracepção hormonal, devido aos efeitos sobre a SHBG e a produção alterada de androgênios dependente de gonadotrofina. É recomendado a suspensão do contraceptivo hormonal por três meses ou mais antes da aferição bioquímica para que se tenha resultados reais (TEED HJ, et al, 2018).

Outro hormônio estudado para detectar SOP é o hormônio anti-mulleriano (AMH), produzido pelas células da granulosa, presentes nos folículos ovarianos. Este hormônio é utilizado na reprodução humana para avaliar a reserva folicular ovariana e têm um valor prognóstico da resposta em um ciclo de estimulação ovariana. Em mulheres diagnosticadas com SOP, os níveis séricos deste hormônio podem checar a valores até três vezes o valor de normalidade. Contudo, não há evidências de que o excesso dele determine a anovulação crônica (NEAGU M e CRISTECECU, 2012; TEED HJ, et al, 2018).

Os níveis séricos de AMH ainda não devem ser usados como uma alternativa para a detecção de SOP ou como um único teste para o diagnóstico de SOP. Há evidências emergentes de que, com a padronização aprimorada de ensaios e níveis de corte ou limiares estabelecidos com base na validação em larga escala em populações de diferentes idades e etnias, AMH pode se tornar um exame mais preciso na na detecção de SOP (NEAGU M e CRISTECECU, 2012; TEED HJ, et al, 2018). 
A infertilidade é uma patologia que compromete o bem-estar e a qualidade de vida de mulheres das mulheres que a enfrentam. As mulheres relatam se sentir anormais e diferentes de todas as outras, por não conseguirem engravidar espontaneamente. Sabidamente, a infertilidade gera sofrimento e tristeza, aumentando a prevalência de depressão nesse grupo de pacientes. Como agravante, algumas culturas e religiões estigmatizam as mulheres que não conseguem engravidar, atribuindo a elas culpa por isso (MOREIRA ST, et al., 2013).

A SOP é apontada como a causa mais frequente de infertilidade por anovulação. As alterações hormonais presentes no comendo do eixo hipotálamo-hipófise-ovariano é o causador dessa alteração. Outrossim, o desequilíbrio entre os fatores extra e intra ovarianos pode agravar o resultado uma foliculogênese anormal (RESENDE LOT, et al., 2010). Em alguns países, como nos Estados Unidos, a SOP é considerada a causa majoritária de infertilidade feminina (FREITAS LA, et al., 2008).

\section{Tratamento}

O tratamento de infertilidade nas pacientes com SOP inclui alterações dos hábitos de vida, com modificações alimentares e inclusão de atividade física, buscando sempre que a paciente esteja dentro do seu índice de massa corporal (IMC) ideal. Essa conduta, muitas vezes, por si só, restaura o equilíbrio hormonal necessário para que ocorra a ovulação (TEED HJ, et al., 2018).

Os tratamentos medicamentosos de primeira linha incluem os indutores da ovulação. No entanto, esses, incluindo letrozol, metformina e citrato de clomifeno, não estão disponíveis para esse uso, em bula, em muitos países. Seu uso off label para indução da ovulação é permitido, porém os profissionais de saúde precisam informar as mulheres e discutir as evidências, possíveis preocupações e efeitos colaterais (TEED HJ, et al., 2018).

Os inibidores da aromatase (Al) são eficazes como agentes indutores da ovulação, incluindo letrozol e anastrozol, com letrozol sendo o mais amplamente utilizado. Esses agentes evitam a conversão de andrógenos em estrógenos induzida por aromatase, que ocorre no ovário. Provalvemente por redução do estrogênio circulante ocorre um aumentam a secreção do hormônio folículo-estimulante (FSH), estimulando o desenvolvimento e a maturação do folículo ovariano (TEED HJ, et al., 2018).

O letrozol deve ser considerado o tratamento farmacológico de primeira linha para a indução da ovulação em mulheres com SOP, com infertilidade anovulatória e nenhum outro fator de infertilidade associado. Quando o letrozol não estiver disponível ou seu uso não for permitido ou o custo for alto para a paciente, os profissionais de saúde podem usar outros agentes de indução da ovulação. Importante informar que o principal motivo desse medicamento ser considerado de primeira linha está no fato de possuir menor risco de gravidez múltipla em comparação com o citrato de clomifeno. As doses habitualmente utilizadas variam de 2,5mg a 7,5mg ao dia por 05 dias (TEED HJ, et al., 2018).

O Citrato de Clomifeno (CC) deve ser usado nas pacientes quando o letrozol não puder ser utilizado. Esse medicamento é um modulador do receptor de estrogênio, podendo atuar como agonista ou antagonista ao hormônio, induzindo a alteração do $\mathrm{GnRH}$, afetando a pulsação e levando ao aumento da liberação de FSH pela hipófise (QUINTERO MM, 2015). Além disso, o Citrato de Clomifeno é um medicamento de baixo custo, de administração oral e com poucos efeitos colaterais, facilitando a aderência ao tratamento (MELO AS, et al., 2015).

A dose inicial do tratamento é de $50 \mathrm{mg}$ por dia, durante 5 dias, começando do segundo ao quinto dia após a menstruação (QUINTERO MM, 2015). Ele deve ser preferível ao uso isolado de metformina para indução da ovulação, em mulheres com IMC maior ou igual a 30m2. As mulheres consideradas resistentes ao CC após dois ciclos, ou com ausência de gestação após seis meses de uso do medicamento, podem se beneficiar da associação com metformina, sobretudo àquelas com obesidade e hiperinsulinemia (MOTTA ELA, et al., 2012).

Segundo estudo de Franik S, et al. (2018), o CC e o letrozol são igualmente seguros quando avaliado a hiperestimulação ovariana e o abortamento espontâneo, no entanto o letrozol possui menores taxas de gestação múltipla e menor risco de hiperestímulo ovariano (SANTANA LF, et al., 2008; TEED HJ, 2018). 
Ainda em relação ao tratamento farmacológico da SOP, as gonadotrofinas podem ser empregadas com a finalidade de gerar uma indução da ovulação. Recomendam-se doses iniciais de FSH de 50 a 75 unidades por dia. Na ausência de resposta após 14 dias, preconiza-se regime step-up, com aumento da dose diária em 12,5 a 37,5 unidades e, depois, a cada 7 dias. O aumento gradual das doses reduz o risco de gravidez múltipla e síndrome de hiperestimulação ovariana (FEBRASGO, 2018).

Algumas evidências relacionam taxas superiores de gravidez clínica, gravidez em curso e nascidos vivos à indução da ovulação com gonadotrofinas quando comparada ao CC (FEBRASGO, 2018). Elas podem ser usadas como agentes farmacológicos de segunda linha em mulheres com SOP que falharam na terapia de indução da ovulação oral de primeira linha e são anovulatórias e inférteis, sem outros fatores de infertilidade. Elas também podem ser consideradas como tratamento de primeira linha, na presença de monitoramento por ultrassom, após aconselhamento sobre custo e risco potencial de gravidez múltipla, em mulheres com SOP com infertilidade anovulatória e nenhum outro fator de infertilidade (TEED HJ, 2018).

Outra opção farmacológica é o Mio-inositol (MI), que é um sensibilizador de insulina e seu uso na SOP visa melhorar os aspectos metabólicos. Estudos sugerem que seu uso implica na diminuição da insulina de jejum e do índice HOMA-IR (índice de resistência insulínica). Além disso, a administração do MI, principalmente quando associado a um estereoisômero do inositol, como o D-chiro-inositol, potencializa os benefícios do seu uso na SOP. Essa administração conjunta resulta na melhoria de alguns parâmetros, tais quais os níveis de LH, testosterona livre, insulina em jejum e no índice HOMA em mulheres que cursam com SOP e obesidade (FEBRASGO, 2018).

No entanto, quanto ao uso do $\mathrm{Ml}$ como droga adjuvante nos desfechos reprodutivos, não há ainda evidências científicas suficientes que sustentem aumento na taxa de nascidos vivos e também não há certeza se pode estar associado a uma queda na taxa de abortamento e diminuição de gestações múltiplas, comparado aos demais tratamentos (FEBRASGO, 2018; SHOWELL MG, et al., 2018, TEED HJ, 2018).

A cirurgia laparoscópica ovariana também é uma alternativa de segunda linha para mulheres com SOP que apresentam resistência ao tratamento com CC. No entanto, o procedimento é eficaz em menos da metade das mulheres, sendo necessária a indução complementar com gonadotrofinas. A indução de ovulação após a cirurgia laparoscópica está associada a altas taxas de ovulação. Contudo, é imprescindível verificar se há indicação de cirurgia, visto que o procedimento possui riscos de destruição do tecido ovariano (SANTANA LF, et al., 2008). Tanto as gonadotrofinas quanto a cirurgia ovariana laparoscópica podem ser usadas em mulheres com SOP com infertilidade anovulatória, resistência ao citrato de clomifeno e nenhum outro fator de infertilidade, após aconselhamento sobre os benefícios e riscos de cada terapia (TEED HJ, 2018).

Para pacientes com SOP, a fertilização in vitro (FIV) é indicada geralmente como terapia de terceira linha quando as terapias de indução de ovulação de primeira ou segunda linha falharam ou também quando há outras causas de infertilidade, tais quais o comprometimento tubário ou casos de infertilidade masculina (FEBRASGO, 2018; TEED HJ, 2018).

O hormônio de estimulação do folículo urinário ou recombinante pode ser usado em mulheres com SOP submetidos a hiperestimulação ovariana controlada para FIV \pm ICSI, com evidência insuficiente para recomendar preparações específicas de hormônio folículo estimulante (FSH). O tratamento com hormônio luteinizante recombinante exógeno não deve ser usado rotineiramente em combinação com terapia com hormônio folículo-estimulante em mulheres com SOP submetidas a hiperestimulação ovariana controlada para FIV \pm ICSI, uma vez que essas pacientes possuem altos nives de LH circulantes (TEED HJ, 2018).

Há alguns riscos ao fazer uma estimulação ovariana para FIV em pacientes com SOP, sendo a síndrome de hiperestimulação ovariana um dos principais. Dessa forma, deve-se atentar para estratégias que visem amenizar estes riscos, entre elas o bloqueio hipofisário com análogos agonistas do $\mathrm{GnRH}$, desencadeamento da maturidade folicular com análogos agonistas do $\mathrm{GnRH}$ e congelamento total de embriões para transferência em ciclo espontâneo posterior. Estudos relatam que a transferência de embriões criopreservados aumenta as chances de sucesso reprodutivo entre mulheres com SOP (FEBRASGO, 2018). 
Medidas não medicamentosas são empregadas. Em um momento inicial são recomendadas medidas dietéticas simples, seguidas de uma perda ponderal em torno de 5-10\% que implicará não apenas em uma melhora estética (hirsutismo, acantose nigrans, acne), mas em toda a qualidade de vida da paciente em questão. A dieta hipocalórica deve ser feita juntamente às atividades físicas intensas e aeróbicas que devem se repetir 3 vezes na semana, com duração de $60 \mathrm{~min}$ cada. Tudo isso melhora não apenas os níveis endócrinos, mas também a parte metabólica que conta com a resistência insulínica e dislipidemia, bem como a regularização dos padrões menstruais (FEBRASGO, 2018).

Por fim, ao considerar a importante influência da hiperinsulinemia na SOP, as drogas sensibilizadoras à insulina, como a metformina, têm sido amplamente recomendadas como alternativa terapêutica de longo prazo para reparação de parâmetros clínicos e endocrinológicos. O funcionamento da metformina ( $\mathrm{N}$ dimetilbiguanida) se baseia em aumentar a utilização de glicose nos tecidos sensíveis à insulina e diminuir a neoglicogênese hepática, fazendo com que os níveis plasmáticos de insulina caiam (ARIE WMY, et al., 2009). Os principais efeitos colaterais associados ao uso da metformina são gastrintestinais como a diarreia, náusea, sabor metálico e desconforto abdominal. Estes efeitos podem ser reduzidos por meio da administração do medicamento durante as refeições e com aumento gradual das doses (ARIE WMY, et al., 2009).

Outro efeito adverso associado à metformina é a acidose lática, bastante temida, entretanto muito rara ( $<0,01-0,08$ casos/mil pacientes por ano, ou seja, 1 a 8 casos em 100 mil usuários), mas que gera mortalidade de $50 \%$. Por esse motivo, é fundamental que a função renal seja previamente avaliada e monitorizada anualmente (ARIE WMY, et al., 2009). Essa medicação deve ser iniciada quando estiver evidente a presença de resistência insulínica associada a SOP, ou quando não houver resposta adequada do ovário aos indutores da ovulação (TEED HJ, 2018).

\section{CONSIDERAÇÕES FINAIS}

O manejo das mulheres inférteis com SOP pode ser complexo, envolvendo várias opções de tratamento. A mudança de estilo de vida, que inclui o acompanhamento nutricional e prática de exercícios físicos deve ser prescrita e incentivada a todas as pacientes. Em associação, podem ser usadas alternativas medicamentosas, que incluem o uso das drogas sensibilizadoras de insulina, indutores da ovulação e demais fármacos. Em casos que, mesmo adotando essas medidas não tenham sucesso, a FIV pode ser indicada. Dessa maneira, ressalta-se a relevância de estudos sobre esse tema por essa patologia ser uma das principais causas de infertilidade, sendo essa busca por atualizações uma forma de possibilitar melhor manejo da doença.

\section{REFERÊNCIAS}

1. ARIE WMY, et al. Síndrome do ovário policístico e metformina: revisão baseada em evidências. Femina, 2009; 37(11): 1-18.

2. CONCHA FC, et al. Epigenética del síndrome de ovario poliquístico. Rev. Méd. Chile, 2017; 145(7): 907-915.

3. Federação Brasileira das Associações de Ginecologia e Obstetrícia (FEBRASGO). Síndrome dos ovários policísticos. São Paulo, 2018.

4. FRANIK S, et al. Aromatase inhibitors for subfertile women with polycystic ovary syndrome. Cochrane Database Syst Rev, 2014; 24(2): 1-122.

5. FREITAS LRA, et al. Uso de metformina em mulheres obesas com Síndrome do Ovário Policístico / Use of metformin in obese women with Polycystic Ovary Syndrome. Rev. ciênc. méd., 2016; 25(2): 87-97.

6. HOFFMAN B, et al. Ginecologia de Willians. $2^{\circ}$ Ed. Porto Alegre: Artmed, 2014.

7. MELO AS, et al. Treatment of infertility in women with polycystic ovary syndrome: approach to clinical practice. Clinics, 2015; 70(11): 765-769.

8. MOREIRA SNT, et al. Qualidade de vida e aspectos psicossociais da síndrome dos ovários policísticos: um estudo quali-quantitativo. Rev. Bras. Ginecol. Obstet., 2013; 35(11):1-8.

9. MOTTA ELA, et al. O uso de sensibilizadores de insulina no tratamento de infertilidade em pacientes com síndrome dos ovários policísticos (SOP). Rev. Bras. Ginecol. Obstet., 2012; 34(3): 99-101.

10. MOURA HHG, et al. Síndrome do Ovário Policístico: abordagem dermatológica. An Bras Dermatol., 2011; 86(1): 111119.

11. NEAGU M, CRISTECU C. Anti-Müllerian hormone--a prognostic marker for metformin therapy efficiency in the treatment of women with infertility and polycystic ovary syndrome. J Med Life, 2012; 5(4): 463-464. 
12. QUINTERO MEM. Síndrome de ovario poliquístico e infertilidad: Opciones de tratamiento. Rev Obstet Ginecol Venez, 2015; 75(4): 269-279.

13. RESENDE LOT, et al. Concentração dos hormônios esteroides no fluido folicular de folículos ovarianos maduros e imaturos de pacientes com síndrome dos ovários policísticos submetidas à fertilização in vitro. Rev. Bras. Ginecol. Obstet., 2010; 32(9): 447-453

14. Rotterdam ESHRE/ASRM-Sponsored PCOS Consensus Workshop Group. Revised 2003 consensus on diagnostic criteria and long-term health risks related to polycystic ovary syndrome. Fertil Steril, 2004; 81(1):19-25.

15. SANTANA LF, et al. Tratamento da infertilidade em mulheres com síndrome dos ovários policísticos. Rev. Bras. Ginecol. Obstet., 2008; 30(4): 201-209.

16. SANTOS RM e ÁLVARES ACM. Revisão de literatura sobre a síndrome do ovário policístico. Rev Inic Cient Ext., 2018; 1(2): 261-265.

17. SHARPE A, et al. Metformin for ovulation induction (excluding gonadotrophins) in women with polycystic ovary syndrome. Cochrane Database of Systematic Reviews, 2019, 12: 1-152.

18. SHOWELL MG, et al. Inositol for subfertile women with polycystic ovary syndrome. Banco de dados Cochrane de revisões sistemáticas, 2018; 12: 1-15.

19. SOUSA RML, et al. Perfil metabólico em mulheres de diferentes índices de massa corporal com síndrome dos ovários policísticos. Rev. Bras. Ginecol. Obstet., 2013; 35(9): 413-420.

20. SPEROFF L, et al. Endocrinologia Ginecológica Clínica e Infertilidade. $5^{\circ}$ Ed. São Paulo: Manole, 1995.

21. TEED HJ, et al. Recommendations from the international evidence-based guideline for the assessment and management of polycystic ovary syndrome. Hum Reprod., 2018;1;33(9):1602-1618.

22. YARAK S, et al. Hiperandrogenismo e pele: síndrome do ovário policístico e resistência periférica à insulina. An. Bras. Dermatol., 2005; 80(4): 395-410. 